\title{
A CORRESPONDENCE CONNECTED WITH A PENCIL OF CURVES OF ORDER $n$
}

\author{
BY A. R. WILLIAMS
}

All curves of order $n$ passing through $\frac{1}{2} n(n+3)-1$ points pass through $\frac{1}{2}(n-1)(n-2)$ other points. Or we may say that the $n^{2}$ base points of a pencil of curves of order $n$ are determined by the number given above. When $\frac{1}{2} n(n+3)-2$ are fixed and another moves on a curve of order $m$, the locus of the remaining $\frac{1}{2} n(n-1)(n-2)$ is a curve of order $m\left(n^{2}-1\right)$ which has a multiple point of order $m n$ at each of the fixed points. The order of the locus is reduced by $n$ for each passage of the given curve through a fixed point.* It is the purpose of this paper to discuss the locus of the remaining base points of a pencil when a number of them are fixed and the others necessary to determine the pencil are taken consecutive on some curve.

We consider first the case when $\frac{1}{2} n(n+3)-3$ points are fixed. Thus for example taking $n=4$, we fix 11 base points of a pencil of quartics and let 2 be consecutive on a line $l$. To find the locus of the remaining three we use the rational quintic surface with a double cubic whose plane sections correspond to the $\infty^{3}$ quartics through the 11 fixed points. To $l$ corresponds on the surface a rational twisted quartic $C_{4}$; and to a pencil of quartics through the 11 points and tangent to $l$ corresponds a pencil of plane sections whose axis is tangent to $C_{4}$. This tangent meets the quintic surface in 3 more points which correspond to the three remaining base points of the pencil of plane quartics. The tangents to $C_{4}$ form a developable of order 6 . Therefore, the plane curve which corresponds to the intersection of the developable with the quintic is of order $6 \times 4$ and has a sextuple point at each of the 11 fixed points. In this locus the line $l$ is counted twice. Hence the locus sought is of order 22. In addition to the singularities at the fixed points

* Milinowski, Journal für Mathematik, vol. 67, p. 263. For $n=3$ we have the Geiser involutorial transformation of order 8. 
it has a number of nodes which correspond to those intersections of the double curve of the developable with the quintic which do not take place on the cuspidal edge $C_{4}$. The developable has 4 stationary tangent planes and a double curve of order 6 . Four tangents to the cuspidal edge meet it again. The points of contact of the stationary planes are ordinary points on both the double curve and the cuspidal edge. A point in which the cuspidal edge is met by a tangent to itself is a cusp on the double curve and an ordinary point on the cuspidal edge. Hence in the present instance the double curve of the developable meets the quintic in $6 \times 5-4-2 \times 4=18$ points which are nodes on the common curve of the developable and the quintic, and which give nodes on the locus we are considering. The double cubic of the quintic surface meets the developable in four points not on the cuspidal edge. These are nodes on their common curve, but do not of course give nodes on the plane locus of order 22. The genus of the latter is, therefore, $210-11 \times 15-18=27$. Since 4 tangents to the cuspidal edge $C_{4}$ meet it again, there are 4 positions of the consecutive pair on $l$ such that one of the remaining 3 falls at a distinct point on $l$. The other 18 intersections of the locus studied with $l$ are self-corresponding. At such a point the pencil of quartics have 3 -point contact; and on the surface $C_{4}$ touches one of the principal tangents to the quintic, and the osculating plane to $C_{4}$ is the tangent plane to the quintic. The plane locus sought and its counterpart on the rational surface must always have the same genus. In this case they are both of order 22 . We have seen that the singularities of the plane curve are the 11 sextuple points (=165 nodes) and 18 nodes corresponding to 18 nodes on the space curve. The further singularities of the latter are the 4 nodes lying on the double cubic and 161 apparent double points. The last number may be verified independently by use of the surface which is the locus of a point the intersection of whose polar planes with respect to the quintic and the developable meets a given line.

Similarly, we may fix $\frac{1}{2} n(n+3)-3$ base points of a pencil of curves of order $n$ and let 2 more be consecutive on a curve $c$ of order $m$ and genus $p$, having $d$ nodes and $k$ cusps apart from the fixed points, and having a multiple point of order $s_{i}$ at a fixed base point $A_{i}$. Then 


$$
d+k+\frac{1}{2} \sum s_{i}\left(s_{i}-1\right)=\frac{1}{2}(m-1)(m-2)-p .
$$

Since only two points are taken consecutive on $c$, the occurrence of a cusp on the latter causes no difficulty. At the cusp we have merely a pencil of curves whose common tangent is the cuspidal tangent. To find the locus of the remaining $\frac{1}{2}(n-1)(n-2)$ points we use the rational surface of order $\frac{1}{2} n(n-3)+3$, whose plane sections correspond to the $\infty^{3} n$-ics through the fixed points. To $c$ corresponds a curve of order $m n-\sum s_{i}$ and genus $p$, having $d$ nodes and $k$ cusps. Hence we have $r=2\left(m n-\sum s_{i}-1\right)-k+2 p$, where $r$ is the order of the developable of its tangents. A curve in 3-space of order $\mu$ and genus $p$, without nodes or cusps, has $2(\mu-2)(\mu-3)+2 p(\mu-6)$ tangents that meet it elsewhere. If an apparent double point is replaced by a node, this number is diminished by 4 ; but if an apparent double point is replaced by a cusp, the reduction is equal to the order $\mu{ }^{*}$ Such tangents meet the curve again in points that are cusps on the double curve of its developable. Replacing $d$ by its value, we have for the number, $\gamma$, of such tangents and points

$$
\begin{aligned}
\gamma=2\left(m n-\sum s_{i}\right)^{2}+\left(m n-\sum s_{i}\right)(2 p & -10-k)+2 \sum s_{i}\left(s_{i}-1\right) \\
& -2 m(m-3)+4 k-8 p+8 .
\end{aligned}
$$

The genus of a plane section of the developable is the same as that of the cuspidal edge. Hence the order, $x$, of the double curve is $\frac{1}{2}(r-1)(r-2)-p-\left(m n-\sum s_{i}\right)$. And the number, $\alpha$, of stationary planes or the number of stationary tangents of a plane section, is

$$
3(r-2)-2\left(m n-\sum s_{i}\right)+6 p .
$$

The order of the locus sought is $r n-2 m$. At a fixed point $A_{i}$ it has a multiple point of order $r-2 s_{i}$. Hence the singularities at the fixed points are equivalent to $\frac{1}{2} \sum\left(r-2 s_{i}\right)\left(r-2 s_{i}-1\right)$ double points. The other nodes are given by the intersections of the double curve of the developable with the rational surface that do not occur on the cuspidal edge. Their number is, therefore, $x\left[\frac{1}{2} n(n-3)+3\right]-\alpha-2 \gamma-4 d-2 k$. The genus is thus found to be

$$
\frac{1}{2}(n-3)\left(5 m n^{2}-5 n \sum s_{i}-6 m-5 n-2 k n\right)+\frac{1}{2} p\left(5 n^{2}-15 n+2\right),
$$

* See Severi, Istituto Lombardo Rendiconti, vol. 21 (1921), p. 251; and Salmon, Solid Geometry, vol. 2, §617. 
which reduces to $p$ when $n=3$. This is necessary since if 6 , or indeed any number, of base points of a pencil of cubics are fixed and enough more to make 8 are taken consecutive on a curve $c$, the locus of the 9th will in general correspond birationally to $c$. In special cases, the correspondence is not birational, but to any point of $c$ will always correspond a single point of the locus of the 9 th. So the genus of the latter will never be greater than the genus of $c{ }^{*}$

The above may immediately be extended to the general case when $\frac{1}{2} n(n+3)-r$ points are fixed. But one difficulty is encountered as shown by the following simple example. Fixing 5 base points of a pencil of cubics and letting 3 be consecutive on a conic $c_{2}$, to find the locus of the 9th we use the rational quartic surface in $S_{4}$ whose hyperplane sections correspond to the $\infty^{4}$ cubics through the fixed points. To $c_{2}$ corresponds a rational sextic $C_{6}$, and to a pencil of cubics through the fixed points and osculating the conic at $P$ correspond a pencil of hyperplane sections having in common the osculating plane to $C_{6}$ at $P^{\prime}$. This osculating plane meets the quartic surface in one more point which corresponds to the 9th base point of the pencil of cubics. The order of the hyperdevelopable of the osculating planes to $C_{6}$ is 12 . Hence the locus sought is of order $12 \times 3-3 \times 2=30$ and has a multiple point of order 12 at each fixed point. There are 18 osculating planes to $C_{6}$ that meet it elsewhere. Hence 42 intersections of the locus of the 9th with $c_{2}$ are self-corresponding, and the others correspond to distinct points on $c_{2}$. The singularities at the fixed points are equivalent to 330 double points; and since the locus must be rational, there are 76 other nodes which correspond to those intersections of the double surface of the hyperdevelopable with the quartic that do not occur on the cuspidal edge. When $n>3$, it would be necessary to determine this number independently. The characteristics of the hyperdevelopable and its double surface, and the order of its triple curve are easily obtained by projection and section. But the double surface contains the cuspidal edge, which of course lies on the rational surface. This makes it difficult in general to find the remaining intersections of the two surfaces. Moreover, the number of

* Picard, Traité d'A nalyse, vol. 2, Chap. XV, §19. 
dimensions of the double variety increases with the number of dimensions of the space in which the configuration is placed. The question thus raised is not of primary importance for the present purpose, and therefore I will not attempt in this paper to give a general formula for the genus of the locus sought when $n>3$ and the number of fixed points is less than $\frac{1}{2} n(n+3)-3$. But in all cases it is easy to find its order, its singularities at the fixed points, and the number of positions of the consecutive set on $c$ such that one of the remaining points of the pencil is a distinct point of $c$.

Thus we fix $\frac{1}{2} n(n+3)-r$ points of a pencil of $n$-ics and let $r-1$ be consecutive on an appropriate curve $c$ which we suppose to have no cusps or multiple points with coincident tangents. To find the locus of the remaining $\frac{1}{2}(n-1)(n-2)$ we use the rational surface of order $\frac{1}{2} n(n-3)+r$ in $r$-space. To $c$ will correspond on the surface a curve $C$ of order $\mu$ determined as above. The hyperdevelopable composed of the $S_{r-2}$ having $(r-1)$-point contact with $C$ meets the rational surface again in the curve which corresponds to the locus that we seek. To find the order of this hyperdevelopable, or the number of such $S_{r-2}$ that meet a general line in $S_{r}$, we project successively on an $S_{r-1}$ and an $S_{r-2}$. It follows immediately that the required number is the number of $S_{r-3}$ (in the projection space $S_{r-2}$ ) that have stationary, that is, $(r-1)$-point, contact with $C^{\prime}$, the projection of $C$ on the $S_{r-2}$. The number of such stationary $S_{r-3}$ is the number of stationary tangents to a plane section of the hyperdevelopable of $C^{\prime}$. This in turn is $3(R-2)-2 K+6 p$, where $R$ is the order of the plane section, $K$ the number of its cusps, and $p$ the genus of $c$, and hence of $C$ and of all curves derived from $C$ by projection and section. But $R$ is the number of $S_{r-4}$ in $S_{r-2}$ that have $(r-3)$-point contact with $C^{\prime}$ and meet a given line. And $K$ is the number of $S_{r-5}$ in $S_{r-2}$ that have $(r-4)$-point contact with $C^{\prime}$ and meet a general plane; that is, the number of $S_{r-5}$ in $S_{r-3}$ that have $(r-4)$-point contact with $C^{\prime \prime}$ and meet a general line in $S_{r-3} . \quad C^{\prime \prime}$ is of course the projection of $C^{\prime}$ on the $S_{r-3}$. Hence if $F_{r}^{\mu}$ denotes the order of the developable of $C$ in $S_{r}$, we have $F_{r}{ }^{\mu}=3\left(F_{r-2}{ }_{-2}-2\right)-2 F_{r}{ }_{-3}+6 p$. We have independently $F_{3}{ }^{\mu}=2(\mu-1)+2 p-k$, where $k$ is the number of cusps on $C$. Hence in general we have 


$$
F_{r}^{\mu}=(r-1)(\mu-r+2)+(r-1)(r-2) p-(r-2) k .
$$

We must suppose that there are no cusps on $c$ for $r>3$. Hence $k=0$. The above result includes the nodes of $C$; that is, there is no difference in this respect between a node and an "apparent" double point. The order of the locus of the $\frac{1}{2}(n-1)(n-2)$ points is therefore $n F_{r}{ }^{\mu}-(r-1) m$, where $m$ is the order of $c$. At a fixed point $A_{i}$ it has a multiple point of order $F_{r}{ }^{\mu}-(r-1) s_{i}$, where $s_{i}$ is the order of $A_{i}$ on $c$. To find the number of positions of the consecutive set on $c$ such that one of the remaining points falls at a distinct point on $c$ we must find the number of $S_{r-2}$ in $S_{r}$ that have $(r-1)$-point contact with $C$ and meet it elsewhere. For a rational curve of order $\mu$ without multiple points in $S_{r}$ (and not contained in a space of fewer dimensions) this number is easily found by the principle of correspondence to be $(r-1)(\mu-r+1)(\mu-r)$. Following the method indicated by Severi in the article referred to, I have found for a similar curve of genus $p$ the number

$$
(r-1)(\mu-r+1)(\mu-r)+\left[\mu(r-1)(r-2)-r(r-1)^{2}\right] p .
$$

Moreover, this number is reduced by $2(r-1)$ when the curve acquires a node without changing its genus.

This method when applied in very simple cases sometimes brings out a fact that is not otherwise obvious. Thus we considered pencils of cubics through 5 fixed points and osculating a conic. If we replaced the conic by a line, the locus of the 9 th point would be the conic determined by the fixed points. This method shows that as the point of osculation describes the line, the 9 th point describes the conic triply. For to the line corresponds on the rational quartic surface in $S_{4}$ a cubic. The 3-space of this cubic meets the quartic surface again in a line which is the image of the conic through the fixed points and a bisecant of the cubic. Through a general point of this line pass three osculating planes to the cubic. The above statement immediately follows.

The UNiversity of CALifornia 